\title{
A New Similarity Measure for Combining Conflicting Evidences
}

\author{
Nadeem Salamat ${ }^{1}$, Nadeem Akhter ${ }^{2, *}$ \\ ${ }^{1}$ Department of Mathematics \& Statistics, Karakoram International University, Gilgit, Pakistan \\ ${ }^{2}$ Department of Computer Science and Information Technology, The Islamia University, Bahawalpur, Pakistan
}

Copyright (C) 2015 by authors, all rights reserved. Authors agree that this article remains permanently

open access under the terms of the Creative Commons Attribution License 4.0 International License

\begin{abstract}
In Dempster-Shafer (DS) theory, multiple information from the distinct information sources are combined to obtain a single Basic Probability Assignment (BPA) function. The well-known combination rule of Dempster-Shafer (DS) provides the weaker solution to the management of conflicting information at the normalization stage. Even this rule fails and provide the counter intuitive results while combining the highly conflicting information. This paper presents a new similarity measure for the combined average methods, where any distance measure between the body of information can be used. The numerical examples provide the promising and better intuitive results.
\end{abstract}

Keywords Similarity Measures, Distance Based Similarity Measure, Combined Averages Rule, Dempster Shafer Combination Rule, Distance Based Combination Rule

\section{Introduction}

Information fusion is the interesting field of research for many researchers in the field of knowledge representation, spatial reasoning, risk analysis, expert systems and information management systems. Many methods have been proposed for information fusion. The Glenn Shafer and Dempster [Shafer, 1976, Dempster, 1967], proposed the possibility theory for the representation and processing information, called the Dempster-Shafer Theory of Belief Functions. In this theory, a combination rule for combining information from multiple sources is also proposed. This theory also deals with uncertainty analysis. It handles the engineering problems including, imprecisely specified distributions, poorly known and unknown correlation between different variables, modeling impression and uncertainty measurement. Unfortunately, the combination rule of evidences, provided by Dempster-Shafer, fails to give realistic results when the information from the sources are conflicting due to any reason. Many extensions in this theory and combination rules have been proposed [Smarandache and Dezert, 2005, Smarandache et al., 2012], and these rules are also extended for the application to in- terval based evidences [Lee and Zhu, 1992].

Lofti. A. Zadeh, in 1984 [Zadeh, 1984], reviewed the book on basic belief theory. He argued some examples where Dempster-Shafer combination rule failed in human intuitional logic and reasoning. Many other such examples can also be find in literature, where the information from multiple sources highly conflict from each other. As the DS- combination rule fails to deal with the conflicting information. This opened a new field for researchers to propose combination rules in the theory of information fusion, when the information from multiple sources highly conflict due to any reason.

The researchers working in this area of research, proposed many algorithms for the management of confecting information. In particular, this problem deals with the redistribution of confecting information with in the possible targets. A simple algorithm for redistribution of conflicting mass are proposed in [Yager, 1986, Smets, 2007], where the yager's approach put the conflicting mass to the ignorance of evidences. The other algorithms include the probabilistic approaches [Smets, 1993, Choi et al., 2009, Denceux, 2006, Joshi et al., 1995], idempotent combination rule [Destercke and Dubois, 2011], algebraic operators [Ali et al., 2012, Lee and Zhu, 1992, Smets, 2007]. The mixing or averaging is proposed in [Sentz and Ferson, 2002, Murphy, 2000], weighted averages are proposed in [Han et al., 2008, Han et al., 2011]. In these methods, the weights of the evidences is defined by the pignistic probability of the evidences. A distance based combination of continuous operators are developed in [Liang-zhou et al., 2005, Attiaoui et al., 2012]. A distance based similarity measure is used to define the averaging operator for the combination of evidences. These measures [ZHANG et al., 2012, Jousselme and Maupin, 2012] use the Jousselme metric for defining averaging rules. Some new metrics are defined in [Djiknavorian et al., 2012], these metrics can also be used for defining weights for information fusion.

We analyse similarity measure defined in [ZHANG et al., 2012, Yong et al., 2004]. The set of discernments is considered as a unit vector, and the Jousselme metric is determined. These measures are defined by a trigonometric function where the jousselme metric is used as angular information. The trigonometric function provide 
the angular information between two vectors. This similarity measure has some limitations and doesn't fulfil all the mathematical properties of a similarity measure. Similarly, for the second similarity measure, it becomes negative for $d>1$. To deal with this problem, we proposed a well define similarity measure defined by the exponential function [Shepard, 1987]. This measure describes the probability that two combination of two events also falls in the space associated with the same response.

This paper is arranged as follows, the next section Section 2 discusses the basic DS-theory and its combination rule. The counter intuitive example of Zadeh is discussed in Section 3. Proposed new similarity measure and related terms are discussed in Section 4. The numerical examples are discussed in Section 5 and Section 6 concludes the paper.

\section{The belief theory}

The theory of evidences and belief also called DempsterShafer theory of evidence, presented by the Glenn Shafer and Dempster in [Shafer, 1976], is described here in a concise manners. This theory is developed to generalize the probability theory by introducing a combination rule for combining the evidences from multiple bodies of evidences. This deals with the uncertain and imprecise knowledge in the expert systems, reasoning and knowledge representation systems. It represents the numerical evidential reasoning. This theory is based on numerical weights of evidence for information fusion.

\subsection{Mass function}

Let $\Omega$ be the space of discernment (events). The mass function ( Basic Probability Assignment (BPA)), is defined from the power set $D$ of discernment to the weight $[0,1]$. A mass function $m$ is defined as

$$
m: 2^{\Omega} \rightarrow[0,1]
$$

The mass function is attached to each independent source of information. The element which has the some quantity of mass is called the focal element. These elements have the following basic properties

$$
m(\emptyset)=0, \quad \sum_{A \subseteq \Omega} m(A)=1
$$

The belief function is defined as

$$
\operatorname{Bel}(A)=\sum_{B \subseteq A} m(B)
$$

and the plausibility function is defined as

$$
P l(A)=\sum_{A \cap B \neq \emptyset} m(B)
$$

It is also a theory of plausible reasoning because it focuses on the fundamental operation of plausible reasoning, namely the combination of evidence.

\subsection{Dempster combination rule}

Let $m_{1}$ and $m_{2}$ be the two frame (evidences), then

$$
\left(m_{1} \oplus m_{2}\right)(A)=m_{12}(A)=\frac{\sum_{B \cap C=A} m_{1}(B) m_{2}(C)}{1-K}
$$

where

$$
K=\sum_{B \cap C=\emptyset} m_{1}(B) m_{2}(C)
$$

It is called Dempster Shafer combination rule. In this theory, $1-K$ is the normalization factor and $K$ captures all the conflicting information. Higher the value of $K$, larger is the disagreement between the bodies providing information. This rule produce some counter intuitive results in case of processing the highly conflicting information, Particularly if the information is totally different. The main problem is to redistribute the confecting information. Following example, elaborates this problem.

\section{Counter intuitive example}

Zadeh [Zadeh, 1984], in his review of book, he quoted an example where, counter intuitive behavior of DScombination rule is marked, which is due to the normalization process. For example, suppose two doctors examine a patient and agree that it suffers from either meningitis $(\mathrm{M})$, contusion $(\mathrm{C})$ or brain tumor $(\mathrm{T})$. Thus frame of discernment is $\Omega=\{M, C, T\}$. Assume that the doctors agree in their low expectation of a tumor, but disagree in likely cause and provide following diagnosis: $m_{1}(M)=0.99, m_{1}(C)=0, m_{1}(T)=0.01$ and $m_{2}(M)=$ $0, m_{2}(C)=0.99, m_{2}(T)=0.01$

The outcome of the DS combination rule of evidences is $m(M)=0, m(C)=0, m(T)=1$, which leads towards an unexpected conclusion. It means that the patient suffers with certainty from brain tumor. This unexpected result arises from the fact that both the bodies of evidence (doctors) agree that the patient most likely does not suffer from tumor but the composition is full of contradiction for the other causes of the disease. The method proposed in this paper provides a reasonable and intuitive results that $m(M)=0.495, m(C)=0.495, m(T)=0.01$. This concludes that there is a most probably an equal chances of the meningitis (M), contusion (C). These results seems to be reasonable and intuitive.

\section{Proposed combination rule}

The similarity measure proposed in [ZHANG et al., 2012], defined as

$$
\operatorname{Sim}_{i, j}=1-d\left(m_{i}, m_{j}\right)
$$

is studied. The example of Zedah is modified a little. Suppose two doctors examine a patient and agree that it suffers from either meningitis (M) or contusion (C). Assume that the doctors disagree in likely cause and provide following diagnosis as $m_{1}(M)=1, m_{1}(C)=0$ and $m_{2}(M)=$ $0, m_{2}(C)=1$

The Jousselme distance [Jousselme and Maupin, 2012] is not exactly the Euclidian distance as they differ by the factor $\frac{1}{2}$. Thus the Euclidian distance between both the bodies is $d\left(m_{1}, m_{2}\right)=1.4243$. In this case, the outcome of the above similarity measure becomes negative, which is counter intuitive behavior of the similarity measure. So, we 
cannot use the Euclidian distance for this similarity measure, secondly. If we use the Jousselme distance, which is $d\left(m_{1}, m_{2}\right)=0.71215$, the value of the similarity measure will become $\operatorname{Sim}=0.28785$, which is not the boundary conditions for a similarity measure.

Now the similarity measure described in [ZHANG et al., 2012], written as

$$
\operatorname{Sim}_{i, j}=\frac{\cos \left(\pi d\left(m_{i}, m_{j}\right)\right)+1}{2}
$$

As $0 \leq \cos (\theta) \leq 1$, this describes that if both the vectors have the opposite values. Then the Joussslme distance is not equal to 1 , so this similarity measure doesn't meet the boundary conditions of a similarity measure. Further, this similarity measure can't be explained geometrically and can't be generalized for every distance measure.

Let $m_{1}$ and $m_{2}$ be the two sources of information having the homogenous frame of discernment. In the distance based similarity measure, the BPAs are considered as a vectors in the space of $n$ dimensional space. The $\sum_{A \in \Omega} A=1$, imposes condition on decision space. As the number of components (targets) increases, the decision space decreases. For combination of BPAs, the following steps are considered

\subsection{Similarity measure}

The Sharped in [Shepard, 1987], proposed that the similarity and distance functions are mutually related via an exponentially function. This measure is represented as

$$
\operatorname{Sim}_{m_{i}, m_{j}}=e^{-D\left(m_{i}, m_{j}\right)}
$$

The measure $\operatorname{Sim}_{i, j}$ defines the similarity between vectors $m_{i}, m_{j}$, and $D\left(m_{i}, m_{j}\right)$ is the Euclidian distance between vectors. This similarity measure is already used for fuzzy decision making in [Williams and Steele, 2002], for generalized distances. The other distances, such as the Jousselme distance proposed in [Jousselme and Maupin, 2012] can also be used. Throughout this paper, the Jousselme distance is used for the comparison point of view with other methods.

\subsection{Weight for the evidence}

The weight for an evidence is defined as

$$
w_{i}=\sum_{j} \operatorname{Sim}_{i, j} \quad \text { where } i \neq j
$$

where $w_{i}$ is the weight for the $i^{t h}$ frame of reference. This represents the un normalized factors, for normalization, we get

$$
W_{i}=\frac{w_{i}}{\sum_{i} w_{i}} \quad \forall i
$$

\subsection{Fusion of evidences}

The combined evidences (the BPA) are determined as

$$
m\left(A_{j}\right)=\sum_{i} W_{i} \times m_{i}\left(A_{j}\right)
$$

for all $j$

\section{Numerical examples}

In this section, the proposed method is compared with the other well-known existing methods. The methods are compared when some evidences are highly conflict from a source due to any reason. For the comparison point of view, the Jousselme distances are used.

Example 1 Let $\Omega=\{A, B, C\}$ be the frame of discernment

\begin{tabular}{|c|c|c|}
\hline Combination Rule & $m_{12}$ & $m_{123}$ \\
\hline Dempster Rule & $\begin{array}{c}m(A)=0.3333 \\
m(B)=0 \\
m(C)=0.6667\end{array}$ & $\begin{array}{l}m(A)=0.6363 \\
m(B)=0 \\
m(C)=0.3637\end{array}$ \\
\hline Yager Rule & $\begin{array}{c}m(A)=0.12 \\
m(B)=0 \\
m(C)=0.24 \\
m(\Omega)=0.64\end{array}$ & $\begin{array}{l}m(A)=0.532 \\
m(B)=0.064 \\
m(C)=0.176 \\
m(\Omega)=0.228\end{array}$ \\
\hline Dubois \& Prade Rule & $\begin{array}{c}m(A)=0.12, \\
m(B)=0, \\
m(C)=0.24, \\
m(A \cup B)=0.02, \\
m(A \cup C)=0.54, \\
m(B \cup C)=0.08\end{array}$ & $\begin{array}{c}m(A)=0.084, \\
m(B)=0, \\
m(C)=0.048 \\
m(A \cup B)=0.028, \\
m(A \cup C)=0.678, \\
m(B \cup C)=0.048, \\
m(\Omega)=0.114\end{array}$ \\
\hline Deng Rule[ZHANG et al., 2012] & $\begin{array}{l}m(A)=0.40 \\
m(B)=0.05 \\
m(C)=0.55\end{array}$ & $\begin{array}{c}m(A)=0.5355 \\
m(B)=0.07499 \\
m(C)=0.38953\end{array}$ \\
\hline Murphy's average rule & $\begin{array}{c}m(A)=0.4 \\
m(B)=0.05 \\
m(C)=0.55\end{array}$ & $\begin{array}{c}m(A)=0.55 \\
m(B)=0.075 \\
m(C)=0.375\end{array}$ \\
\hline Smet's Rule & $\begin{array}{c}m(A)=0.12 \\
m(B)=0 \\
m(C)=0.24 \\
m(\emptyset)=0.64\end{array}$ & $\begin{array}{c}m(A)=0.084 \\
m(B)=0 \\
m(C)=0.048 \\
m(\emptyset)=0.868\end{array}$ \\
\hline Tazid Ali Rule & $\begin{array}{l}m(A)=0.3896 \\
m(B)=0.0398 \\
m(C)=0.5706\end{array}$ & $\begin{array}{l}m(A)=0.3446 \\
m(B)=0.4116 \\
m(C)=0.2438\end{array}$ \\
\hline Proposed Rule & $\begin{array}{c}m(A)=0.4, m(B)=0.05 \\
m(C)=0.55\end{array}$ & $\begin{array}{c}m(A)=0.52153 \\
m(B)=0.071566, \\
m(C)=0.40816\end{array}$ \\
\hline
\end{tabular}
and the corresponding basic probability assignments (bpa) from three different independent sources are:

$$
\begin{aligned}
& \text { - } m_{1}(A)=0.6, m_{1}(B)=0.1, m_{1}(C)=0.3 \\
& \text { - } m_{2}(A)=0.2, m_{2}(B)=0.0, m_{2}(C)=0.8 \\
& \text { - } m_{3}(A)=0.7, m_{3}(B)=0.1, m_{3}(C)=0.2
\end{aligned}
$$

Table 1. Results for Different Combination Rules of Evidences

In this example, the information from the source $m_{2}$ conflict from the other sources. The computational results from the methods are shown in the table (1). The output results from two sources are same for the Murphy's average rule, Deng Rule and proposed method. This is because all these methods works as averaging for combining information from two sources. When the information for third sources is combined. we get the improvement for the event $A$ and $C$. An improvement for the output of discernment $A$ can be observed, where the information from the source are more precise and less weight is given to the conflicting information.

Example 2 Let $\Omega=\{A, B, C\}$ be the frame of be the frame of discernment and the corresponding basic probability assignments (bpa) from four different independent sources are:

- $m_{1}(A)=0.98, m_{1}(B)=0.01, m_{1}(C)=0.01$

- $m_{2}(A)=0.00, m_{2}(B)=0.01, m_{2}(C)=0.99$

- $m_{3}(A)=0.90, m_{3}(B)=0.01, m_{3}(C)=0.09$

- $m_{4}(A)=0.90, m_{4}(B)=0.01, m_{4}(C)=0.09$ 
Table 2. Results for Different Combination Rules of Evidences

\begin{tabular}{|c|c|c|c|}
\hline Combination Rule & $m_{12}$ & $m_{123}$ & $m_{1234}$ \\
\hline & $m(A)=0, m(B)=0.01$, & $m(A)=0$, & $m(A)=0$, \\
Dempster Rule & $m(C)=0.99$ & $m(B)=0.0011$, & $m(B)=0.0001$, \\
& $m(A)=0$, & $m(C)=0.9989$ & $m(C)=0.9999$ \\
\hline & $m(B)=0.0001$, & $m(B)=0.8908$, & $m(A)=0.81$, \\
Yager Rule & $m(C)=0.0099$ & $m(C)=0.09$, & $m(B)=0.0002$, \\
& $m(\Omega)=0.9898$ & $m(\Omega)=0.01$ & $m(C)=0.009$, \\
& $m(A)=0$, & $m(A)=0, m(B)=0$, & $m(A)=0, m(B)=0$, \\
& $m(B)=0.0001$, & $m(C)=0.0089$, & $m(C)=0.0008$, \\
& $m(C)=0.0099$, & $m(A \cup B)=0.009$ & $m(A \cup B)=0.0082$ \\
Dubois \& Prade Rule & $m(A \cup B)=0.0098$ & $m(A \cup C)=0.9694$, & $m(A \cup C)=0.9677$, \\
& $m(A \cup C)=0.9702$, & $m(B \cup C)=0.002$ & $m(B \cup C)=0.0003$ \\
& $m(B \cup C)=0.01$ & $m(\Omega)=0.01958$ & $m(\Omega)=0.0319$ \\
\hline & $m(A)=0.4898$, & $m(A)=0.998299$, & $m(A)=0.998426$, \\
& $m(B)=0.0002$, & $m(B)=0.000001$, & $m(B)=0.00000001$, \\
& $m(C)=0.51$ & $m(C)=0.17$ & $m(C)=0.0001457$ \\
\hline \multirow{3}{*}{ Deng Rule } & $m(A)=0.49$, & $m(A)=0.695$, & $m(A)=0.797$, \\
& $m(B)=0.01$, & $m(B)=0.01$, & $m(B)=0.01$, \\
& $m(C)=0.5$ & $m(C)=0.295$ & $m(C)=0.193$ \\
\hline \multirow{3}{*}{ Smet's Rule } & $m(A)=0$, & $m(A)=0$, & $m(A)=0$, \\
& $m(B)=0.0001$, & $m(B)=0$, & $m(B)=0$, \\
& $m(C)=0.0099$ & $m(C)=0.0009$ & $m(C)=0.0001$ \\
& $m(\emptyset)=0.99$ & $m(\emptyset)=0.9991$ & $m(\emptyset)=0.9999$ \\
\hline \multirow{2}{*}{ Tazid Ali Rule } & $m(A)=0.4924$, & $m(A)=0.7016$, & $m(A)=0.8075$, \\
& $m(B)=0.0051$, & $m(B)=0.0059$, & $m(B)=0.0068$, \\
& $m(C)=0.5025$ & $m(C)=0.2925$ & $m(C)=0.1857$ \\
\hline \multirow{2}{*}{ Proposed Rule } & $m(A)=0.49$, & $m(A)=0.705938$, & $m(A)=0.77427$, \\
& $m(B)=0.01$, & $m(B)=0.009999$, & $m(B)=0.01005$, \\
& $m(C)=0.50$ & $m(C)=0.284033$ & $m(C)=0.22087$ \\
\hline
\end{tabular}

In this example, we consider the case, where the information from the source $m_{1}$ and $m_{2}$ conflict for target $A$ and $C$ whereas the information for the target $B$ are stable.

The computed results are presented in table (2). The results shows that, the results are almost similar in proposed method and already existing techniques ([Murphy, 2000, Ali et al., 2012, Yong et al., 2004]) for combining information from two sources. When we add the information from the third source, the information for the target A are improved, which are conflicting due to many causes from the source $m_{2}$. After the addition of information from the source $m_{4}$, the proposed method provides the less weight to the event $A$ much weight to event $C$, as compared to the other methods.

The information for the target $B$ is remains same for each source. The simulated results from the proposed method are also very close to the information provided from each source. This seems to be reasonable. The information for the target $C$ is highly conflicting from source $m_{1}$ and $m_{2}$, so the computed results are closer to the results by the method proposed in [Murphy, 2000, Ali et al., 2012, Yong et al., 2004]).

Example 3 Let $\Omega=\{A, B, C\}$ be the frame of discernment and the corresponding basic probability assignments (bpa) from five independent sources are:

$$
\begin{aligned}
& \text { - } m_{1}(A)=0.5, m_{1}(B)=0.2, m_{1}(C)=0.3 \\
& \text { - } m_{2}(A)=0.9, m_{2}(B)=0.00, m_{2}(C)=0.1 \\
& \text { - } m_{3}(A)=0.55, m_{3}(B)=0.1, m_{3}(C)=0.35 \\
& \text { - } m_{4}(A)=0.55, m_{4}(B)=0.1, m_{4}(C)=0.35 \\
& \text { - } m_{5}(A)=0.6, m_{5}(B)=0.1, m_{5}(C)=0.3
\end{aligned}
$$

where the information for the discernment from the source $m_{1}$ and source $m_{2}$ varies where as the source $m_{3}$ and $m_{4}$ have the same information and source $m_{5}$ provides us a slightly different bpa. In the following table, we compare the numerical results for different methods.

In this table (3), columns represent the composition of information from the sources as mentioned in first row. It is evident that the classical Dempster rule, Yager rule and

\begin{tabular}{|c|c|c|c|c|}
\hline Combination Ruld & $m_{12}$ & $m_{123}$ & $m_{1234}$ & $m_{12345}$ \\
\hline Dempster Rule & $\begin{array}{c}m(A)=0.9375 \\
m(B)=0 \\
m(C)=0.0625\end{array}$ & $\begin{array}{c}m(A)=0.9593 \\
m(B)=0 \\
m(C)=0.04069\end{array}$ & $\begin{array}{c}m(A)=0.9736 \\
m(B)=0 \\
m(C)=0.02629\end{array}$ & $\begin{array}{c}m(A)=0.9867 \\
m(B)=0 \\
m(C)=0.01332\end{array}$ \\
\hline Yager Rule & $\begin{array}{c}m(A)=0.45 \\
m(B)=0 \\
m(C)=0.03 \\
m(\Omega)=0.52\end{array}$ & $\begin{array}{c}m(A)=0.5335, \\
m(B)=0.052, \\
m(C)=0.1925, \\
m(\Omega)=0.222\end{array}$ & $\begin{array}{l}m(A)=0.4155 \\
m(B)=0.0275 \\
m(C)=0.1459, \\
m(\Omega)=0.3989\end{array}$ & $\begin{array}{l}m(A)=0.4887 \\
m(B)=0.04265 \\
m(C)=0.1634 \\
m(\Omega)=0.29306\end{array}$ \\
\hline Dubois \& Prade Rule & $\begin{array}{c}m(A)=0.45, \\
m(B)=0, \\
m(C)=0.03, \\
m(A \cup B)=0.18 \\
m(A \cup C)=0.32 \\
m(B \cup C)=0.02\end{array}$ & $\begin{array}{c}m(A)=0.2475, \\
m(B)=0, \\
m(C)=0.0105, \\
m(A \cup B)=0.162 \\
m(A \cup C)=0.462, \\
m(B \cup C)=0.012 \\
m(\Omega)=0.106\end{array}$ & $\begin{array}{c}m(A)=0.1361 \\
m(B)=0, \\
m(C)=0.0037 \\
m(A \cup B)=0.13 \\
m(A \cup C)=0.508 \\
m(B \cup C)=0.00645 \\
m(\Omega)=0.7402\end{array}$ & $\begin{array}{c}m(A)=0.08166, \\
m(B)=0, \\
m(C)=0.001, \\
m(A \cup B)=0.1046 \\
m(A \cup C)=0.5002, \\
m(B \cup C)=0.0029 \\
m(\Omega)=0.8338\end{array}$ \\
\hline Deng Rule & $\begin{array}{l}m(A)=0.7 \\
m(B)=0.1 \\
m(C)=0.2\end{array}$ & $\begin{array}{c}m(A)=0.619597 \\
m(B)=0.116185 \\
m(C)=0.2642\end{array}$ & $\begin{array}{c}m(A)=0.590 \\
m(B)=0.11518, \\
m(C)=0.29481\end{array}$ & $\begin{array}{l}m(A)=0.59799 \\
m(B)=0.10895 \\
m(C)=0.29306\end{array}$ \\
\hline Iurphy's Average Rule & $\begin{array}{l}m(A)=0.7 \\
m(B)=0.1, \\
m(C)=0.2\end{array}$ & $\begin{array}{c}m(A)=0.625 \\
m(B)=0.1 \\
m(C)=0.275\end{array}$ & $\begin{array}{c}m(A)=0.587 \\
m(B)=0.1 \\
m(C)=0.3125\end{array}$ & $\begin{array}{c}m(A)=0.594 \\
m(B)=0.1 \\
m(C)=0.306\end{array}$ \\
\hline Smet's Rule & $\begin{array}{c}m(A)=0.45, \\
m(B)=0, \\
m(C)=0.03 \\
m(\emptyset)=0.52\end{array}$ & $\begin{array}{c}m(A)=0.247 \\
m(B)=0, \\
m(C)=0.010 \\
m(\emptyset)=0.74\end{array}$ & $\begin{array}{c}m(A)=0.136, \\
m(B)=0, \\
m(C)=0.004 \\
m(\emptyset)=0.86\end{array}$ & $\begin{array}{c}m(A)=0.082, \\
m(B)=0 \\
m(C)=0.001 \\
m(\emptyset)=0.917\end{array}$ \\
\hline Tazid Ali Rule & 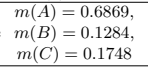 & $\begin{array}{l}m(A)=0.6427 \\
m(B)=0.1022, \\
m(C)=0.2551\end{array}$ & $\begin{array}{c}m(A)=0.6144, \\
m(B)=0.09024, \\
m(C)=0.2953\end{array}$ & $\begin{array}{l}m(A)=0.62538 \\
m(B)=0.08503 \\
m(C)=0.28959\end{array}$ \\
\hline Proposed Rule & $\begin{array}{l}m(A)=0.7, \\
m(B)=0.1, \\
m(C)=0.2\end{array}$ & $\begin{array}{l}m(A)=0.63852, \\
m(B)=0.10439, \\
m(C)=0.25709\end{array}$ & $\begin{array}{l}m(A)=0.612378, \\
m(B)=0.10413, \\
m(C)=0.283472\end{array}$ & $\begin{array}{l}m(A)=0.609835 \\
m(B)=0.10413 \\
m(C)=0.286938\end{array}$ \\
\hline
\end{tabular}
Dubois \& Prade rule does not represent the actual situation for the event $B$, where the information are conflicting. Although the information from the sources $m_{3}, m_{4}$ and $m_{5}$
Table 3. Results for Different Combination Rules of Evidences

make clear the situation. The results for the other methods are very close and there is a small fluctuation in the out puts for the events due to the information provided by the source $m_{2}$, which highly deviate from the information provided from other sources.

\section{Conclusion}

Dempster's combination rule provides the weaker solution to problem of combining the conflicting information from multiple information sources at the normalization stage. The moving averages provides a better solution to this problems.

The distance between BPAs is generalized and a new similarity measure is used. This new similarity measure satisfy all the properties of similarity measure. This new measure works well the generalized distance and results are improved. The numerical examples shows the new proposed similarity measure and the generalized distances are more suitable for combining the conflicting information.

\section{Acknowledgements}

The authors are grateful to the anonymous reviewers for their valuable comments during the review process. Their comments help us a lot to improve the text of the paper.

\section{REFERENCES}

[Shafer, 1976] Shafer, G. (1976). A mathematical Theory of Evidence. Princeton Univ. Press, Princeton New Jersey.

[Dempster, 1967] Dempster, A. (1967). Upper and lower probabilities induced by a multi-valued mapping. Annual Mathematics and Statistics, 38(4):325-339.

[Smarandache and Dezert, 2005] Smarandache, F. and Dezert, J. (2005). A Simple Proportional Conflict Redistribution Rule. JAIF Journal, pages 1-36.

[Smarandache et al., 2012] Smarandache, F., Han, D., and Martin, A. (2012). Comparative study of contradiction measures in the theory of belief functions. In Information Fusion ( $F U$ SION), 2012 15th International Conference on, pages 271277. 
[Lee and Zhu, 1992] Lee, E. and Zhu, Q. (1992). An interval dempster-shafer approach. Computers \& Mathematics with Applications, 24(7):89 - 95.

[Zadeh, 1984] Zadeh, L. A. (1984). A Review of Books: A Mathematical Theory of Evidence. GLENN SHAFER. Princeton University Press, Princeton, NJ, 1976. AI Magazine, 5(3):8183.

[Yager, 1986] Yager, R. R. (1986). Arithmetic and other operations on dempster-shafer structures. International Journal of ManMachine Studies, 25:357-366.

[Smets, 2007] Smets, P. (2007). Analyzing the combination of conflicting belief functions. Information Fusion, 8(4):387 - 412.

[Smets, 1993] Smets, P. (1993). Belief functions: The disjunctive rule of combination and the generalized bayesian theorem. In ternational Journal of Approximate Reasoning, 9(1):1 - 35.

[Choi et al., 2009] Choi, H., Katake, A., Choi, S., Kang, Y., and Choe, Y. (2009). Probabilistic combination of multiple evidence. In Neural Information Processing, volume 5863, pages 302-311.

[Denceux, 2006] Denceux, T. (2006). The cautious rule of combination for belief functions and some extensions. In Information Fusion, 2006 9th International Conference on, pages $1-8$.

[Joshi et al., 1995] Joshi, A. V., Sahasrabudhe, S. C., and Shankar, K. (1995). Sensitivity of combination schemes under conflicting conditions and a new method. In SBIA, pages 39-48.

[Destercke and Dubois, 2011] Destercke, S. and Dubois, D. (2011). Idempotent conjunctive combination of belief functions: Extending the minimum rule of possibility theory . Information Sciences, 181(18):3925 - 3945.

[Ali et al., 2012] Ali, T., Dutta, P., and Boruah, H. (2012). A New Combination Rule for Conflict Problem of Dempster-Shafer Evidence Theory. International Journal of Energy, Information and Communications, 3(1).

[Sentz and Ferson, 2002] Sentz, K. and Ferson, S. (2002). Combination of evidence in dempster-shafer theory. Technical report SAND2002-0835, SANDIA Tech.

[Murphy, 2000] Murphy, C. K. (2000). Combining belief functions when evidence conflicts. Decis. Support Syst., 29(1):1-9.
[Han et al., 2008] Han, D., Han, C., and Yang, Y. (2008). A modified evidence combination approach based on ambiguity measure. In Information Fusion, 2008 11th International Conference on, pages 1-6.

[Han et al., 2011] Han, D., Dezert, J., Han, C., and Yang, Y. (2011). New dissimilarity measures in evidence theory. In Information Fusion (FUSION), 2011 Proceedings of the 14th International Conference on, pages 1-7.

[Liang-zhou et al., 2005] Liang-zhou, C., Wen-kang, S., Yong, D., and Zhen-fu, Z. (2005). A new fusion approach based on distance of evidences. Journal of Zhejiang University SCIENCE A, 6(5):476-482.

[Attiaoui et al., 2012] Attiaoui, D., Dor, P.-E., Martin, A., and Yaghlane, B. B. (2012). A distance between continuous belief functions. In Hllermeier, E., Link, S., Fober, T., and Seeger, B., editors, Scalable Uncertainty Management, volume 7520 of Lecture Notes in Computer Science, pages 194-205. Springer Berlin Heidelberg.

[ZHANG et al., 2012] ZHANG, H., LI, Y., and DENG, Y. (2012). A New Method of Combining Conflict Evidences. Journal of Computational Information Systems, 8(8):3421-3427.

[Jousselme and Maupin, 2012] Jousselme, A.-L. and Maupin, P. (2012). Distances in evidence theory: Comprehensive survey and generalizations. International Journal of Approximate Reasoning, 53(2):118-145.

[Djiknavorian et al., 2012] Djiknavorian, P., Grenier, D., and Valin, P. (2012). New metrics between bodies of evidences. Journal of Emerging Technologies in Web Intelligence.

[Yong et al., 2004] Yong, D., WenKang, S., ZhenFu, Z., and Qi, L. (2004). Combining belief functions based on distance of evidence. Decis. Support Syst., 38(3):489-493.

[Shepard, 1987] Shepard, R. (1987). Toward a universal law of generalization for psychological science. Science, 237(4820):1317-1323.

[Williams and Steele, 2002] Williams, J. and Steele, N. (2002). Difference, distance and similarity as a basis for fuzzy decision support based on prototypical decision classes. Fuzzy Sets Syst., 131(1):35-46. 